\title{
Interventional Strategies in early Atherosclerosis
}

\section{Strategie di intervento nella Aterosclerosi precoce}

\author{
Marinella Gattone, Pantaleo Giannuzzi
}

\begin{abstract}
Interventional Strategies in early Atherosclerosis. M. Gattone, P. Giannuzzi.

Atherosclerosis remains clinically mute for a long time and frequently manifests itself with an acute cardiovascular event; therefore, the possibility to detect the disease in a subclinical phase and to reduce or reverse its progression is an issue of relevance. Non-invasive diagnostic procedures such as B-mode ultrasonography of carotid intima-media thickness (CIMT), electron beam computed tomography (EBCT) and magnetic resonance angiography (MRA) allow to identify atherosclerotic disease in its early phases, to evaluate the disease progression and monitor the effects of interventions.

In recent years, several therapeutic strategies have been adopted over time to slow early atherosclerosis in asymptomatic individuals at intermediate/high cardiovascular risk.

Prospective trials employing multifactorial non-pharmacological interventions (diet, exercise, smoking cessation)
\end{abstract}

\begin{abstract}
have demonstrated a favorable effect on progression of atherosclerosis. Hence lifestyle modification may be an effective therapeutic strategy to be adopted as a first step and a highly cost-effective intervention in a preclinical setting involving a large number of individuals. Drugs should be considered as a second step or should be associated to further reduce the risk in individuals at high probability of future events, who require more intensive interventions.

Reducing low-density lipoprotein levels, blood pressure and platelet aggregation seems to be the most effective intervention in these subjects, whereas the treatment of emerging coronary risk factors, e.g. oxidative stress, inflammatory activation and infections has not produced the expected protective effect.

Keywords: early atherosclerosis, non-invasive imaging, cardiovascular prevention.
\end{abstract}

Monaldi Arch Chest Dis 2006; 66: 54-62.

IRCCS Fondazione “Salvatore Maugeri”, Istituto Scientifico di Veruno (NO); Divisione di Cardiologia Riabilitativa

Corresponding author: Marinella Gattone, Divisione di Cardiologia Riabilitativa IRCCS Fondazione "Salvatore Maugeri", Istituto Scientifico di Veruno; Via per Revislate 13, I-28010 Veruno (NO) Italy; E-mail address: mgattone@fsm.it

Atherosclerosis is a continuous process which remains clinically mute for a long time and frequently manifests with an acute cardiovascular event. Thus the possibility to detect the disease in a subclinical phase and slow or reverse its progression is certainly an issue of relevance warranting investigation.

In recent years non-invasive imaging techniques have been found to be useful tools for detecting atherosclerotic disease in its early phases. B-mode ultrasound measurement of carotid intima-media thickness (CIMT) is the most widely employed method, but also electron beam computed tomography (EBCT) to identify coronary artery calcifications and magnetic resonance angiography (MRA) are increasingly used. Echographic measurement of CIMT is widely used in clinical studies as a surrogate marker for atherosclerotic disease and, in prospective studies, increased CIMT is predictive of cardiovascular events [1, 2]; serial EBCT allows to follow up the progression of coronary calcium, detecting changes in atherosclerotic plaque burden over time; MRA is a promising noninvasive technique for the longitudinal study of large atherosclerotic arteries [3].

These diagnostic procedures permit a more accurate assessment of subjects at intermediate to high risk of cardiovascular disease, as classified according to traditional risk stratification [4]. Furthermore, they constitute a reliable methodology for evaluating the effects of therapeutic intervention on the pro- gression of subclinical atherosclerotic disease, as demonstrated by a number of recent studies in which both pharmacological and non pharmacological strategies were adopted.

\section{Pharmacological interventions}

\section{Statins}

In the mid '90s several studies demonstrated a decrease in CIMT induced by statin therapy in patients with, or at high risk of, coronary artery disease [5-7]. Later, in a retrospective study on asymptomatic subjects, Callister et al. [8] found that HMGCoA reductase inhibitors could reduce the volume of calcified plaque detected by EBCT in the coronary arteries. After at least one year of therapy, a slower progression of the disease in treated patients was observed at any level of LDL cholesterol reached, while the control group showed a net increase in coronary calcium. A stabilization or real decrease of coronary plaque volume was evident only in patients in whom LDL cholesterol levels of less than $120 \mathrm{mg}$ per deciliter had been achieved.

Using MRA Corti et al. [2] evaluated wall thickness, vessel wall area and lumen area of 44 aortic and 32 carotid artery plaques in 21 asymptomatic hypercholesterolemic subjects treated with simvastatin. Lipid lowering therapy significantly reduced plaque size, vessel wall area and wall thickness, regardless of location, after at least 12 months of treat- 
ment. A further favorable vessel wall remodeling of aortic and carotid plaques was evident at 18 and 24 months.

Since initial studies indicated that the effects of statin therapy could be dependent on the level of LDL$\mathrm{C}$ reduction, further observations aimed to verify if an aggressive cholesterol lowering in the early phases of the disease could be more effective in reducing atherosclerosis progression than a moderate one.

In the ASAP study [9], 325 patients with familial hypercholesterolemia were randomized to receive atorvastatin $80 \mathrm{mg}$ or simvastatin $40 \mathrm{mg}$ daily. Changes in mean CIMT were assessed by quantitative B-mode ultrasound. After 24 months, patients treated with high dose atorvastatin had a greater cholesterol reduction than those treated with moderate dose simvastatin. CIMT significantly decreased in the atorvastatin group, whereas it increased in the simvastatin group. Similar results were found in the ARBITER study [10]. In 161 high risk patients (46\% with known cardiovascular disease), after 12 months of treatment, atorvastatin $80 \mathrm{mg} /$ day induced a progressive reduction of CIMT, whereas CIMT remained unchanged in the group treated with pravastatin $40 \mathrm{mg}$.

The results of these latter studies are consistent with those of the REVERSAL study [11]. In patients with advanced coronary artery disease atorvastatin $80 \mathrm{mg}$ daily was more effective than pravastatin 40 $\mathrm{mg}$ in reducing coronary atherosclerotic plaque volume, evaluated with intravascular ultrasound.

High dose atorvastatin lowered LDL cholesterol levels more than did pravastatin $(-48.5 \%$ versus $-27.2 \%$ in ARBITER); nevertheless the anti-atherosclerotic effect of atorvastatin was superior to that of pravastatin at each cholesterol level reached, suggesting that a more pronounced anti-inflammatory action could have influenced the slower progression of the disease. Indeed in the REVERSAL study atorvastatin lowered PCR levels more than pravastatin (-36.4\% vs $-5.2 \%)$.

Previous studies have shown that statin are beneficial in the early stages of the disease even at moderate doses, while recent trials seem to indicate that a more intensive lipid lowering may be necessary to stabilize or reverse the atherosclerotic lesions. Furthermore, the additive property of some molecules might enhance their effectiveness, independently of cholesterol reduction.

\section{Long chain $n-3$ polyunsaturated fatty acids (n-3 LC-PUFA)}

Epidemiological data indicate that in populations exposed to high nutritional intake of n-3 PUFA the incidence of atherosclerosis is low. Observational studies support the epidemiological data, showing the efficacy of n-3 PUFA in primary prevention of coronary artery disease: the Nurses Health Study [12], carried out on more than 100,000 healthy women, found that fish intake at least twice a week was associated to a 30\% reduction of cardiovascular risk. In the Physicians' Health Study [13], a marked reduction in sudden death in healthy male physicians with elevated whole-blood n-3 fatty acid concentrations was found. A case-control study, nested in the
Cardiovascular Health Study on elderly subjects [14], showed a significant inverse relation between serum n-3 PUFA concentrations and risk of fatal ischemic heart disease. Only the Health Professionals follow-up [15], conducted on more than 40,000 healthy subjects, was negative: no significant correlation between a fish rich diet and risk of coronary artery disease was found, although some benefit was evident comparing those who ate at least a small quantity of fish with those who ate none at all.

Observations in subjects with subclinical atherosclerosis are few in number. A Japanese cross-sectional study showed a significant inverse relation between dietary intake of EPA and DHA and presence of carotid atherosclerosis in 1,920 subjects aged over 40 years [16], while negative findings came from a more limited clinical trial in which 223 patients with coronary artery disease were treated with dietary supplementation of n-3 PUFA (1.65 g/day) for two years, without showing a significant effect on progression of atherosclerosis in carotid arteries [17].

Interesting is the study by Thies et al. [18], who evaluated the anti-inflammatory and anti-thrombotic properties of long chain n-3 and n-6 PUFA in a randomized controlled double-blind trial on 162 patients awaiting preventive carotid endoarterectomy. Patients were treated daily with $6 \mathrm{~g}$ of fish-oil (n-3), sunflower oil (n-6) or control oil (blend of palm and soybean oils) until surgery to evaluate the degree of stability of surgically removed plaques. In fish-oil treated patients, carotid plaque lipids had higher concentrations of n-3 PUFA in proportion to the duration of the treatment. Furthermore, in fish-oil treated patients a greater proportion of plaques with a well-formed fibrous cap, without thrombosis and a lower quantity of thin-cap inflamed atheromas were observed than in the other oil groups. n-3 PUFA reduced the production of chemoattractants and the infiltration of macrophages, leading to plaque stabilization and reducing the vulnerability of atherosclerotic lesions, mainly through the modification of their lipidic composition.

Observational studies indicate a clinical benefit of dietary n-3 PUFA in primary prevention, and the demonstration of a plaque stabilization obtainable with n-3 PUFA in a relatively short time is promising. In addition, a cardioprotective effect of long chain n-3 PUFA has been demonstrated in secondary prevention intervention trials such as the GISSI-Prevenzione study [19], in which pharmacological n-3 PUFA supplements were associated with a significant reduction of subsequent major events. Also in the GISSI-Prevenzione study survival curves began to diverge after about 60 days of treatment, supporting the hypothesis of an early effect of plaque stabilization in n-3 PUFA treated patients.

\section{Aspirin}

Primary prevention of cardiovascular events with aspirin has been studied on more than 50,000 subjects. While a significant reduction of acute cardiac events has been found, only a minor effect on all-cause mortality and no effect on stroke is evident [20]. Indeed in low risk individuals, the benefits of aspirin may be counterbalanced by an increase of 
adverse effects, in particular gastrointestinal bleeding and hemorrhagic stroke. However, as the Antiplatelet Trialists meta-analysis [21] shows, in patients with established CAD the risk/benefit ratio is more favorable: at doses between 75 and $150 \mathrm{mg}$ daily the drug significantly reduces the incidence of cardiovascular fatal and nonfatal events and of nonfatal stroke, and the benefit substantially outweighs the risk of major bleeding.

Aspirin has been evaluated in preclinical phases of atherosclerotic disease in just a few studies with small cohorts and conflicting results. Delker [22] did not find any effect of 100 to $300 \mathrm{mg} /$ day of ASA on CIMT progression in 54 subjects with coronary risk factors, while Kodama [23] found both ASA and ticlopidine to have some efficacy in 148 diabetics.

Nervertheless, considering that some form of asymptomatic atherosclerosis may be present in subjects with an elevated concentration of cardiovascular risk factors, there are several primary prevention studies indicating that aspirin might be effective in reducing cardiovascular events in these individuals. In fact, in the Nurses Health Study [24] the beneficial effect of aspirin on thrombotic strokes was more evident in women who were older, hypertensive or smokers. In other prevention studies, aspirin reduced more the relative risk of myocardial infarction in subjects over 70 years than in younger ones (HOT, 25 PPP, 26). In the Physician Health Study [27] subjects with diabetes had a greater benefit from ASA than non diabetics. Antiplatelet therapy was effective against non-fatal cardiac events in males at high risk for ischemic heart disease in the Thrombosis Prevention Trial [28]. This agent reduced major cardiovascular events also in the hypertensive patients of the Hypertension Optimal Treatment Study [25].

Further demonstrations come from the Primary Prevention Project (PPP) [26], a large trial specifically designed to evaluate the effect of low dose aspirin in the prevention of cardiovascular events in high risk people "as routinely identified and treated in clinical practice". More than 2,000 males and females presenting at least one major risk factor, such as old age ( $\geq 65$ years), systolic blood pressure $\geq 160$ $\mathrm{mmHg}$ or diastolic blood pressure $\geq 95 \mathrm{mmHg}$, total blood cholesterol $\geq 6.4 \mathrm{mmol} / \mathrm{L}$, fasting venous plasma glucose concentration $\geq 7.8 \mathrm{mmol} / \mathrm{L}$, body mass index $\geq 30 \mathrm{~kg} / \mathrm{m}^{2}$ and family history of myocardial infarction before 55 years in at least one parent or sibling, were randomized to receive $100 \mathrm{mg}$ /day of aspirin or placebo, in addition to the conventional treatment of presented risk factors.

The trial was stopped after a mean follow-up of 3-6 years because the benefit of treatment was already evident. Cardiovascular death was reduced by $44 \%$ and total cardiovascular events, including CV deaths, non-fatal myocardial infarction, non-fatal stroke, TIA, angina pectoris, peripheral-artery disease and revascularization procedures, were reduced by $23 \%$ in subjects taking aspirin. However, hemorrhagic stroke increased by $27 \%$, particularly subarachnoid hemorrhage associated with aspirin therapy in uncontrolled hypertension, as seen in the Physician Health Study.

The results of primary prevention trials on high risk subjects indicate that aspirin is a suitable and ef- fective preventive strategy, although some matter of concern exists regarding the possibility of hemorrhagic events in poorly controlled hypertensive patients.

\section{Beta-blockers}

Some long term studies on the anti-atherosclerotic effects of beta blockers in early stages of atherosclerosis using ultrasound techniques have been carried out in the last few years.

The Beta-Blocker Cholesterol-Lowering Asymptomatic Plaque Study (BCAPS) [29] tested low-dose metoprolol succinate controlled release/extended release (CR/XL) (25 mg once a day) vs placebo in 793 subjects with asymptomatic carotid plaques. After 18 months of treatment the drug significantly reduced carotid plaque thickness and the effect was still evident 3 years later. Incidence of fatal and non fatal cardiovascular events was also lower in the treatment group.

The ELVA trial (Effects of Long-Term treatment with Metoprolol CR/XL on Surrogate Variables for Atherosclerotic Disease) [30] enrolled hypercholesterolemic patients who had plaques in the carotid artery region (or IMT max in the common carotid artery $>1 \mathrm{~mm}$ ). Patients were first treated with lipidlowering therapy and then randomized to metoprolol or placebo to test if beta blockers could provide additional benefit concerning atherosclerotic progression with respect to that given by statins. Results of the ELVA study showed that, in hypercholesterolemic patients already on lipid-lowering therapy, metoprolol further slowed carotid atherosclerosis progression.

Anti-atherosclerotic effects of atenolol were compared to those of lacidipine in the ELSA study [31] and to those of losartan in the LAARS [32] and LIFE [33] trials. Atenolol resulted less effective than lacidipin in reducing CIMT in ELSA, while the results of LAARS and LIFE were conflicting: atenolol was similar to losartan in the first study, but inferior in the latter, which had a longer follow-up period.

The autonomic nervous system certainly plays a role in the development and progression of atherosclerosis. The studies with beta-blockers indicate that they may have an additional, protective effect, particularly in subjects already treated with lipid lowering agents [34]. Less evident is the benefit in studies which directly compared beta-blockers with calcium antagonists or angiotensin II receptor blockers, suggesting that a worse lipid profile with beta-blockers could influence the outcome.

\section{Angiotensin-converting enzyme inhibitors (ACE-I) and angiotensin II receptor blockers (ARB)}

Hypertension increases IMT of the arterial wall promoting development and progression of atherosclerosis. In a large number of hypertensive subjects, the anti-atherosclerotic effects of ACE-I and ARB even in the early phases of the disease have been documented. The multicenter PROTECT trial [35] on 800 hypertensive middle-aged patients found that perindopril was more effective than hy- 
drochlorothiazide on carotid and femoral IMT progression. Similarly, in the PHYLLIS study [36] fosinopril $20 \mathrm{mg}$ daily slowed the carotid atherosclerosis progression in hypertensive hypercholesterolemic patients, whereas IMT significantly progressed in the hydrochlorothiazide-treated group, although blood pressure reduction was similar in both groups.

A substudy of HOPE, the SECURE study [37], evaluated the effects of long term treatment with ramipril (2.5 to $10 \mathrm{mg}$ day) in subjects over 55 years, with vascular disease or diabetes and at least one other cardiovascular risk factor. After 4.5 years, progression of carotid atherosclerosis was significantly slowed in the ramipril group. Even in this study, the effects remained significant after adjusting for systolic and diastolic blood pressure changes, indicating that the benefit was independent of a purely hypotensive action.

Other positive studies were the ELVERA trial [38], in elderly newly diagnosed hypertensive patients, in whom lisinopril 10 to $20 \mathrm{mg}$ daily significantly decreased carotid IMT with the maximum effectiveness after one year of treatment.

The Losartan Vascular Regression Study (LAARS) [32] tested losartan $50 \mathrm{mg}$ once daily in patients with essential hypertension, showing a significant reduction in common CIMT over 24 months. In a substudy of LIFE [33], losartan reduced the hypertrophy of common carotid arteries and incidence of stroke in hypertensive patients.

Hypertension is associated to high prevalence of increased intima/media thickness of the arterial wall and experimental evidence suggests that activation of the renin-angiotensin-aldosterone system plays an important role in atherosclerosis. Studies with ACE-I and ARB in subclinical phases of the disease have shown a positive effect of these agents on atherosclerosis progression, chiefly through blood pressure reduction, but also through the restoration of the endothelium dependent vasodilatation.

\section{Calcium channel-blockers}

In 1990 the INTACT [39] study was the first to demonstrate that a calcium channel-blocker, nifedipine, could slow angiographic progression of coronary artery disease and reduce newly formed coronary lesions. Specifically designed studies later tested the hypothesis that calcium channel blockers play a role in early atherosclerosis. The INSIGHT trial [40] confirmed the anti-atherosclerotic efficacy of nifedipine. In 439 hypertensive patients studied by carotid ultrasonography this agent was superior to co-amilozide, although blood pressure decreased similarly with both treatments. Positive results were also obtained with isradipine (MIDAS study) [41] and verapamil (VHAS study) [42], both more effective than diuretics on carotid progression.

Amlodipine was studied in the PREVENT study [43], which included 825 males and females with angiographic documented coronary artery disease with a baseline diameter stenosis of $30 \%, 377$ of whom underwent B mode carotid ultrasonography to evaluate the effect of amlodipine versus placebo on progression of early coronary and carotid athero- sclerosis. No effect on coronary lesions was observed but the 36-month carotid progression was significantly reduced with amlodipine. The different sensitivity of two instrumental techniques in detecting evolution of the arterial disease and the possible different hypotensive effect obtained with amlodipine on the carotid and coronary circulation are possible explanations accounting for this discrepancy.

Amlodipine was compared to lisinopril, with similar results, in the ELVERA trial [38], conducted on elderly hypertensive patients, whereas in the study of Stanton et al. [44] amlodipine was more effective than lisinopril in reducing CIMT.

The largest and longest trial carried out with calcium antagonists is the ELSA study (European Lacidipine Study on Atherosclerosis) [45]. More than 2,000 patients were enrolled and followed up for 4 years, comparing lacidipine to atenolol. Despite a lesser decrease in blood pressure compared with the beta-blocker, the reduction of asymptomatic carotid progression and of the number of plaques per patient was greater in the lacidipine treated patients. In this latter group also a better lipid profile was evident: HDL cholesterol was higher and triglycerides were lower than in atenolol patients.

A positive action of the calcium antagonists on atherosclerosis progression independent of the hypotensive effect has been evidenced by a series of studies; the hypothesized mechanisms for the antiatherosclerotic effect are anti-oxidative and anti-inflammatory action, restoration of endothelial function and the absence of a negative effect on lipid profile.

\section{Antioxidants}

The oxidation of lipoproteins is a central process in the pathogenesis and progression of atherosclerosis and, hence, antioxidants such as vitamins $\mathrm{E}$ and $\mathrm{C}$ and beta-carotene are expected to be effective in the prevention and stabilization of the atherosclerotic lesions. Epidemiological data support this hypothesis, showing a lower incidence of ischemic heart disease and stroke in populations with a high intake of fruit and vegetables, but it has been difficult to identify which components of the diet are the most protective.

When consumption of each single antioxidant was evaluated, some positive evidence was found only for Vitamin E (Nurses Health Study and Health Professional Follow up study) [46, 47]. Disappointing results also emerged from large randomized prospective trials such as the Primary Prevention Project (PPP) [26] and the Heart Protection Study (HPS) [48]. No effects on any cardiovascular event were observed in PPP, carried out on more than 2,000 high risk subjects with at least one major risk factor, randomized to receive $300 \mathrm{mg} /$ day of vitamin E. Similarly negative were the results of the Heart Protection Study, in which the combination of 600 $\mathrm{mg}$ of vitamin E, $250 \mathrm{mg}$ of vitamin $\mathrm{C}$ and $20 \mathrm{mg}$ of beta-carotene daily had no effect on the subsequent incidence of cardiovascular event in a large population of males and females.

Hypothesizing that a benefit of anti-oxidation could be more evident in earlier stages of the dis- 
ease, some studies have been conducted in this setting. The observational CUDAS [49] evaluated the association between dietary vitamin intake and fasting plasma levels of vitamins A,C and E, lycopene, beta-carotene and carotid IMT in more than 1,000 subjects. A decrease in mean CIMT with increasing quartile of dietary vitamin $\mathrm{E}$ intake was seen only in men. In women an inverse association with the lycopene intake was found. None of the other agents considered influenced CIMT.

The randomized prospective ASAP study [50] showed some positive findings. After 6-year supplementation with vitamin E 136 IU and vitamin C 250 mg twice daily, common carotid artery IMT slowed down in 520 subjects of both sexes, aged 45 to 69 years, with serum cholesterol $\geq 193 \mathrm{mg} / \mathrm{dl}$. The benefit was more evident in males $(-33 \%, \mathrm{p}=0.02)$ than in females $(-14 \%$, NS), in smokers than in non smokers, in subjects with low baseline plasma vitamin $\mathrm{C}$ and in those with plaques in the common carotid artery, suggesting that anti-oxidant therapy could have some positive effect when employed in subjects at high risk for oxidative stress. In a similar population, however, the SECURE trial [37] did not confirm the results of ASAP: in fact, vitamin E 400 IU daily did not influence CIMT progression after 4.5 years of follow-up in males and females aged $>55$ years at high risk of cardiovascular disease.

A lipid-lowering agent with antioxidant and antiinflammatory properties, probucol, was tested in the FAST study [51]. At a dosage of $500 \mathrm{mg}$ daily probucol was compared to pravastatin $10 \mathrm{mg}$ daily and to diet alone (control) in 246 asymptomatic hypercholesterolemic patients, in whom measurement of carotid IMT was performed at the beginning of the study and after 2 years of follow-up. Both drugs reduced CIMT by the same amount $(-13.9 \%)$, whereas in the control group it increased $(+23 \%)$. The IMT reduction with probucol was the same as that with pravastatin, although probucol was more active in cholesterol lowering, suggesting that part of the probucol effect on atherosclerosis progression is independent of its lipidlowering action. Moreover the CIMT reduction occurred earlier than with pravastatin.

The contradictory findings of trials on the whole, even considering some positive results, do not permit to identify a clear protective effect of antioxidants in early phases of atherosclerosis.

\section{Antibiotics}

In the last twenty years, many observations have suggested a possible association between chronic infections and cardiovascular disease. Particularly intracellular pathogens, including Chlamydia pneumoniae, Helicobacter pylori, Cytomegalovirus and Herpes virus A, sometimes directly detected in atherosclerotic lesions, have been associated to the disease. Furthermore, prospective studies showed that subjects with serologic evidence of previous infections, particularly multiple infections, are at high risk of cardiovascular disease and cross-sectional observations evidenced that patients with known coronary artery disease had more frequently seropositivity or elevated antibody titres for one or more of these pathogens.
How chronic infections might promote cardiovascular disease is still far from clear. One hypothesis is that intracellular pathogens have a direct effect on the vascular wall, causing a series of reactions stimulating the onset of atherosclerotic lesions, or that they act indirectly, mediated by a systemic inflammatory activation, through the increase of acute phase reactants such as leukocytes, fibrinogen, or Creactive protein.

Trials on antibiotic treatment conducted in early phases of atherosclerosis have been encouraging [52]. In two studies abdominal aortic aneurysm growth was reduced by antibiotic treatment (roxithromycin or doxycycline) $[53,54]$. Sander et al. [55] evaluated IMT progression of the common carotid artery in 272 patients with ischemic stroke. CIMT and Chlamydia pneumoniae (Cp) antibodies were measured at least three years before the start of antibiotic therapy. The authors found that patients who were $\mathrm{Cp}$-seropositive $(\operatorname{IgG} \geq 1: 64$ or $\operatorname{IgA} \geq 1: 16)$ had a more pronounced progression of carotid atherosclerosis than seronegative patients. All patients were randomized to receive roxithromycin $150 \mathrm{mg}$ twice daily for 30 days. After two additional years of follow-up, antibiotic treatment significantly decreased carotid progression in patients with antibodies to $\mathrm{Cp}$ whereas it did not demonstrate any effect in seronegative patients. The frequency of clinical end-points was not influenced by the antibiotic therapy. In this study, roxithromycin, a macrolide with anti-inflammatory activity, did not change the prevalence of seropositivity during the follow-up, but significantly decreased C-reactive protein levels, elevated in Cp positive patients, suggesting that the mechanism by which $\mathrm{Cp}$ influences atherosclerosis progression may be its promotion of the inflammatory response in the vascular wall. Prolonging the follow-up, after two further years Sander and coworkers found that progression in Cp positive patients climbed back to similar values as before treatment, suggesting that a short-term antibiotic therapy has only a transient effect on atherosclerosis progression in this population [56].

The results of these observations, in patients with early atherosclerosis, are in line with the conflicting findings of antibiotic trials in secondary prevention of CAD. Indeed, the infective hypothesis of atherosclerosis has raised high expectations, but results coming from trials on antibiotic treatment are not definitive. The relative small size of the studies and the short course of the treatment are the main causes cited as possible explanations. One further hypothesis is that a complete eradication of intracellular pathogens may be difficult and that longer treatment periods could be necessary.

\section{Lifestyle modification}

There is evidence that lifestyle modification, including dietary changes, weight control, increased physical activity and smoking cessation, greatly influence development and progression of atherosclerosis through the improvement of lipoprotein profile, blood pressure, glucose levels, haemostatic balance and inflammatory response. In addition, a series of angiographic trials have shown that lifestyle 
changes may slow or reverse coronary lesions in subjects with known ischemic heart disease.

In the last few years, some experimental studies on non-pharmacological interventions have been reported also in a preclinical setting. The impact of dietary changes on carotid IMT progression was evaluated in the MARS [2] and FAST [51] studies. In these trials, the control groups received only dietetic indications, while intervention groups were also treated with lipid-lowering drugs (lovastatin or pravastatin/probucol). Low-fat, low-cholesterol diet alone was unable to reduce CIMT in subjects with early atherosclerosis; in fact CIMT consistently increased during the follow-up period in both trials, although in FAST a significant reduction of LDL-C level had been achieved.

Results of observational studies on physical activity and progression of carotid atherosclerosis are equivocal. The ARIC study [57] did not find leisure time activity to have any effect, but it found workplace activity to be protective. Also in the British Regional Heart Study [58] no association between participation in sporting activity and CIMT was found. Self reported leisure time activity was not associated with carotid atherosclerosis progression in a Finnish study [59], but an inverse relation with the frequency of vigorous activities and physical fitness was found. Similarly the Los Angeles Atherosclerosis Study [60], in 500 middle aged asymptomatic males and females, found that progression of carotid atherosclerosis was inversely related to the amount of physical activity during leisure but not during work, the benefit increasing in proportion to the activity level. Anti-atherogenic benefits were already evident among sedentary persons who adopted a moderately active lifestyle.

In the prospective randomized intervention DNASCO Study (DNA polymorphism and Carotid Atherosclerosis) [61] progressive low to moderate aerobic exercise was able to attenuate CIMT progression only in men not taking statins, suggesting that anti-atherosclerotic effects of statins could minimize or mask the effects of exercise.

Encouraging results came from intervention trials in which lifestyle changes were more extensive. Re-examining the placebo group of the MARS study, Markus et al. [62], found that when lifestyle modification included not only low-fat diet but also smoking cessation, alcohol reduction and weight loss, progression of early preintrusive carotid atherosclerosis could be reduced.

A multi-factorial preventive intervention was carried out by Okada et al. [63] in a large prospective trial in a suburban Japanese population. The 596 hypercholesterolemic subjects enrolled were asked to adopt NCEP step 2 diet, to do aerobic physical exercise ( $>30 \mathrm{~min}$ three times a week), to stop smoking and reduce excessive alcohol intake; 159 of them were also treated with low-dose simvastatin (5 $\mathrm{mg}$ per day). After 2 years, lifestyle modification had significantly inhibited or reduced progression of atherosclerosis in carotid arteries. CIMT progression was more influenced by the changes in serum cholesterol level than by the changes in other lifestyle related risk factors. The benefit was evident even when TC level, although reduced, remained above the level usually recommended, in contrast with the finding of the mono-factorial FAST trial, suggesting that even a moderate reduction in TC level may contribute to slow the disease progression, when associated to other lifestyle interventions.

Finally, the Women's Healthy Lifestyle Project (WHLP) [64] further shows that lifestyle intervention (diet/exercise) could slow menopause related carotid atherosclerosis progression.

Differences in methodology, population characteristics, type and intensity of interventions, and follow-up length may at least partially explain dissimilar results of the studies in which lifestyle has been evaluated.

In summary, prospective trials based on multifactorial interventions have shown more efficacy in slowing carotid atherosclerosis progression in asymptomatic subjects than mono-factorial, frequently observational, studies. When diet was employed as an isolated intervention, results were quite disappointing. Nevertheless, it should be considered that control groups of pharmacological trials cannot be considered an ideal setting to test a treatment: nutritional advice may not have been as strong, or diet compliance as adequate, as in specifically designed dietary intervention trials. Similarly, the different methods of physical activity measurement, frequently self-reported, and spontaneous variations in intensity and duration of physical activity across the follow-up period might account for the variability in findings in the different studies. Nevertheless, the benefit evident in some studies when energy expenditure levels were quite elevated, seems to indicate a protective role of physical activity even in preclinical atherosclerosis.

\section{Conclusions}

Non-invasive imaging techniques such as Bmode ultrasonography of carotid intima-media thickness, electron beam computed tomography to detect coronary calcium and magnetic resonance angiography allow to identify atherosclerosis in its earlier phases and to evaluate the disease progression and compare the benefits of interventions [4]. Furthermore, these surrogate measures may permit to predict the clinical outcome; a recent meta-analysis on more than 90,000 patients at risk of or with established CHD and treated with statins showed that the incidence of stroke diminishes proportionally to the reduction in CIMT progression, giving a further validation of carotid ultrasonography use as surrogate marker [65].

Several therapeutic strategies have been adopted over time to slow atherosclerosis in asymptomatic individuals at elevated cardiovascular risk. As Greenland and coworkers pointed out, this category certainly includes subjects with high serum cholesterol and blood pressure levels and those at elevated risk (absolute risk $>20 \%$ at ten years) on the basis of traditional risk stratification, but it may also include subjects who fall within the intermediate risk zone, whose risk status could advance from intermediate to high through a further risk stratification with non invasive testing [4]. 
Pharmacological interventions have been tested in numerous studies, whereas lifestyle modification has been relatively less evaluated. Nevertheless, prospective trials adopting multifactorial non-pharmacological interventions (diet/ exercise/smoking cessation) have consistently demonstrated lower carotid IMT progression. In addition, the evidence that lifestyle intervention is effective in secondary prevention of CAD is strong, as clinical and angiographic trials have demonstrated. Hence, in a preclinical setting in which a large number of individuals are implicated, lifestyle modification should be adopted as the first step of intervention and the most cost-effective.

Drugs should be considered as a second step or should be associated when the aim is to reduce the risk in subjects at higher probability of a future event, who require more intensive interventions. Reducing low-density lipoprotein levels, blood pressure and platelet aggregation seems to be the most effective intervention in these subjects.

Several instrumental trials have shown that intensive therapy with synthetic statins are more effective than standard-dose therapy in early atherosclerosis. Nevertheless, some prospective clinical studies employing standard doses of statins in high risk individuals have obtained a significant reduction of clinical end-points. Thus the possibility that even small reductions of atherosclerosis progression, detected by non invasive imagine techniques, may have a measurable clinical benefit is real. In fact, in the ASCOT-LLA study [66] on middle-aged and elderly patients at elevated cardiovascular risk profile, lipid lowering therapy with fixed low-dose atorvastatin $(10 \mathrm{mg} /$ daily) reduced fatal CHD and non-fatal myocardial infarction by $36 \%$ after 3 years. Furthermore, therapy with standard doses of simvastatin showed a $25 \%$ reduction in the incidence of coronary events and a 50\% decrease in carotid endarterectomy in the HPS [67]. Thus, considering the clinical efficacy of standard-dose therapy and the elevated cost and enhanced possibility of side effects of the aggressive therapy, the use of high dosage statin therapy in a primary prevention setting seems not recommendable.

A series of other pharmacological agents such as ACE inhibitors, angiotensin II antagonists, calcium channel-blockers and beta-blockers have been widely employed to slow atherosclerosis progression by means mainly of the improvement of the hypertensive component. In some trials a direct comparison was made between calcium antagonists and ACE inhibitors or angiotensin II antagonists with substantially similar positive outcomes. However, when compared with beta-blocker atenolol, both lacidipin (ELSA) [31] and losartan (LIFE) [32] showed greater efficacy concerning CIMT progression. The benefit of these pharmacological agents appears to go beyond a pure hypotensive action, since, in several observations, also with diuretics a similar control on blood pressure was reached, but diuretics were ineffective on CIMT. The different outcome has been attributed to a favorable effect on lipid profile, the property of restoring endothelium-dependent vasodilatation and reduction of circulating markers of oxidative stress.
Although the specific effect of antiplatelet agents on carotid IMT progression has been poorly studied, the results of primary prevention trials indicate a more evident benefit of aspirin in subjects at higher risk. In particular, the large Primary Prevention Project [26] shows that low-dose aspirin, given in addition to treatment of specific risk factors, reduces the frequency of major fatal and non fatal events in subjects with one or more major coronary risk factor, although the possibility of hemorrhagic events in poorly controlled hypertensive patients exists [27].

The studies with n-3 PUFA in carotid atherosclerosis show a promising capacity to obtain plaque stabilization in a relatively short time. However the elevated dose of n-3 PUFA daily (6 g) required to obtain an evident instrumental benefit [18] and the absence, until now, of large prospective intervention trials exploring the possible benefit of lower doses of this treatment in a preclinical phase of the atherosclerotic disease limit the clinical applicability of this therapy.

Emerging coronary risk factors, e.g. oxidative stress, inflammatory activation and infections, have raised high expectations regarding new, different types of pharmacological treatment such as antioxidants and antibiotics. However, despite the positive results of some interesting studies, supplementation with antioxidant in the early phases of atherosclerosis has not produced the protective effect hypothesized on the basis of dietary observational studies. The only exception was probucol, which also has lipid-lowering and anti-inflammatory properties.

Finally, antibiotic therapy is nowadays hardly proposable in the clinical setting to slow down atherosclerosis progression, although the short term results obtained in subjects with seropositivity to $\mathrm{Cp}$ are quite promising.

\section{References}

1. Bots ML, Hoes AW, Koudstaal PJ, et al. Common carotid intima-media thickness and risk of stroke and myocardial infarction; the Rotterdam Study. Circulation 1997; 96: 1432-7.

2. Chambless LE, Heiss G, Folsom AR, et al. Association of coronary heart disease incidence with carotid arterial wall thickness and major risk factors: the Atherosclerotic Risk in Communities (ARIC) Study, 1987-1993. Am J Epidemiol 1997; 146: 483-94.

3. Corti R, Fuster V, Fayad ZA, et al. Lipid lowering by simvastatin induces regression of human atherosclerotic lesions. Two years' follow-up by high-resolution noninvasive magnetic resonance imaging. Circulation 2002; 106: 2884-87.

4. Greenland P, Smith SC, Grundy SM. Improving coronary heart disease risk assessment in asymptomatic people. Role of traditional risk factors and non invasive cardiovascular tests. Circulation 2001; 104: 1863-67.

5. Furberg CD, Adams HP Jr, Applegate WB, et al. Effect of lovastatin on early carotid atherosclerosis and cardiovascular events. Asymptomatic Carotid artery progression Study (ACAPS). Circulation 1994; 90: 1679-87.

6. Hodis NH, Mack WJ, LaBree L, et al. Reduction in carotid arterial wall thickness using lovastatin and dietary therapy. A Randomized, controlled, clinical trial. Ann Int Med 1996; 124: 548-556.

7. Crouse JR, Bryington RP, Bond MG, et al. Pravastatin, lipids and atherosclerosis in the carotid arteries (PLAC II). Am J Cardiol 1995; 75: 455-59. 
8. Callister TQ, Raggi P, Cooil B, Lippolis NJ, Russo DJ. Effect of HMG-CoA reductase inhibitors on coronary artery disease as assessed by electron beam computed tomography. N Engl J Med 1998; 339: 1972-8.

9. Smilde TJ, van Wissen S, Wollersheim H, Trip MD, Kastelein JJP, Stalenhoef AFH. Effect of aggressive versus conventional lipid lowering on atherosclerosis progression in familial hypercholesterolaemia (ASAP): a prospective, randomised, double-blind trial. Lancet 2001; 357: $577-81$.

10. Taylor AJ, Kent SM, Flaherty PJ, Coyle LC, Markwood TT, Vernalis MN. ARBITER: Arterial Biology for the Investigation of the Treatment Effects of Reducing Cholesterol. A randomized trial comparing the effects of atorvastatin and pravastatin on carotid intima medial thickness. Circulation 2002; 106: 2055-60.

11. Nissen SE. Effect of intensive lipid-lowering on progression of coronary atherosclerosis: evidence for an early benefit from the Reversal of Atherosclerosis with Aggressive Lipid Lowering. Am J Cardiol 2005; 96: 61 F-68 F.

12. Hu FB, Bronner L, Willett WC, et al. Fish and omega-3 fatty acids intake and risk of coronary artery disease in women. J Am Med Assoc 2002; 2871815-21.

13. Albert CM, Campos H, Stampfer MJ, et al. Blood levels of long-chain n-3 fatty acids and the risk of sudden death. N Engl J Med 2002; 346: 1113-8.

14. Lemaitre RN, King IB, Mozaffarian D, et al. n-3 polyunsaturated fatty acids, fatal ischemic heart disease, and nonfatal myocardial infarction in older adults: the Cardiovascular Health Study. Am J Clin Nutr 2003; 77: 320-5.

15. Ascherio A, Rimm EB, Stampfer MJ. Dietary intake of marine n-3 fatty acids, fish intake, and the risk of coronary disease among men. $N$ Engl J Med 1995; 332: 977-82.

16. Hino A, Adachi H, Toyomasu K, et al. Very long chain n3 fatty acids intake and carotid atherosclerosis: an epidemiological study evaluated by ultrasonography. Atherosclerosis 2004; 176: 145-9.

17. Angerer P, Kothny W, Stork S, von Schacky C. Effect of dietary supplementation with omega 3 fatty acids on progression of atherosclerosis in carotid arteries. Cardiovasc Res 2002; 54: 183-90.

18. Thies F, Garry JMC, Yaqoob P, et al. Association of n-3 polyunsaturated fatty acids with stability of atherosclerotic plaques: a randomized trial. Lancet 2003; 361: 447-85.

19. GISSI-prevenzione Investigators. Dietary supplementation with $n-3$ polyunsaturated fatty acids and vitamin $\mathrm{E}$ in 11324 patients with myocardial infarction: results of the GISSI-Prevenzione trial. Lancet 1999; 354: 447-55.

20. Metha P. Aspirin in the prophylaxis of coronary artery disease. Curr Opin Cardiol 2002; 17: 552-558.

21. Antithrombotic Trialists' Collaboration. Collaborative meta-analysis of randomized trials of antiplatelet therapy for prevention of death, myocardial infarction. BMJ 2002: 324: 71-86.

22. Ascherio A, Rimm EB, Stampfer MJ, Giovannucci EL, Willett WC. Dietary intake of marine n-3 fatty acids, fish intake, and the risk of coronary disease among men. $N$ Engl J Med 1995; 332: 977-82.

23. Kodama M, Yamasaki Y, Sakamoto K, et al. Antiplatelet drugs attenuate progression of carotid intima-media thickness in subjects with type 2 diabetes. Thromb Res 2000; 97: 239-45.

24. Iso H, Hennekens CH, Stampfer MJ, et al. Prospective study of aspirin use and risk of stroke in women. Stroke 1999; 30: 1764-71.

25. Hansson L, Zanchetti A, Carruthers SG, et al. Effects of intensive blood-pressure lowering and low dose aspirin in patients with hypertension: principal results of the Hypertension Optimal Treatment (HOT) randomized trial. HOT Study Group. Lancet 1998; 351: 1755-62.

26. de Gaetano G. Low-dose aspirin and vitamin $\mathrm{E}$ in people at cardiovascular risk: a randomized trial in general prac- tice. Collaborative Group of the Primary Prevention Project (PPP). Lancet 2001; 357: 89-95.

27. Steering Committee of the Physicians' Health Study Research Group. Final report on the aspirin component of the ongoing Physician Health Study. N Engl J Med 1989; 321: 129-35.

28. Thrombosis Prevention Trial: randomised trial of low-intensity oral anticoagulation with warfarin and low-dose aspirin in the primary prevention of ischemic heart disease in men at increased risk. The Medical Research Council's General Practice Research Framework. Lancet 1998; 351: 233-41.

29. Hedblad B, Wickstrand J, Janzon L, Wedel H, Berglund G. Low-dose metoprolol $\mathrm{CR} / \mathrm{XL}$ and fluvastastin slow progression of carotid intima-media thickness. Main results from the beta-Blocker Cholesterol-Lowering Asymptomatic Plaque Study (BCAPS). Circulation 2001; 103: 1721-26.

30. Wiklund O, Hulthe J, Wickstrand J, Schmidt C, Olofsson S-O, Bondjers G. Effect of controlled release/extended release metoprolol on carotid intima-media thickness in patients with hypercholesterolemia: a 3 year randomized study. Stroke 2002; 33: 572-77.

31. Zanchetti A, Bond MG, Hennig M, et al. Calcium antagonist lacidipine slows down progression of asymptomatic carotid atherosclerosis. Principal results of the European Lacidipine Study on Atherosclerosis (ELSA), a randomized, double blind, long-term trial. Circulation 2002; 106: 2422-27.

32. Ludwig M, Stapff M, Ribeiro A, et al. Comparison of the effects of losartan and atenolol on common carotid artery intima-media thickness in patients with hypertension: results of a 2-year, double blind, randomized, controlled study. Clin Ther 2002; 24: 1175-93.

33. Olsen $\mathrm{MH}$, Wachtell $\mathrm{K}$, Neland $\mathrm{K}$, et al. Losartan but not atenolol reduce carotid artery hypertrophy in essential hypertension. A LIFE substudy. Blood Pressure 2005; 14: $177-83$

34. Wickstrand J, Berglund G, Hedblad B, Hulthe J. Antiatherosclerotic effects of beta-blockers. Am J Cardiol 2003; 91 (suppl): 25H-29H.

35. Stumpe KO, Ludwig M, Heagerty AM, et al. Vascular wall thickness in hypertension: the Perindopril Regression of Vascular Thickening European community Trial: PROTECT. Am J Cardiol 1995; 76: 50E-54E.

36. Zanchetti A, Crepaldi G, Bond MG, et al. Different effects of antihypertensive regimens based on fosinopril or hydrochlorothiazide with or without lipid lowering by pravastatin on progression of asymptomatic carotid atherosclerosis: principal results of PHYLLIS- a randomized double-blind trial. Stroke 2004; 35: 2807-12

37. Lonn EM, Yusuf S, Dzavik V, et al. Effects of ramipril and vitamin $\mathrm{E}$ on atherosclerosis. The study to evaluate carotid ultrasound changes in patients treated with ramipril and vitamin E (SECURE). Circulation 2001; 103: 919-25.

38. Terpstra WF, May JF, Smit AJ, Graeff PA, Meyboom-de Jong B, Crijns HJ. Effects of amlodipine and lisinopril on intima-media thickness in previously untreated, elderly hypertensive patients (the ELVERA trial). $J$ Hypertension 2004; 22: 1309-16.

39. Lichtlen PR, Hugenholtz PG, Rafflenbeul W, et al. on behalf of the INTACT Group Investigators. Retardation of angiographic progression of coronary artery disease by nifedipine: results of the International Nifedipine Trial Of Antiatherosclerotic Therapy. Lancet 1990; 335: 11091113.

40. Simon A, Garlepy J, Moyse D, Levenson J. Differential effects of nifedipine and co-amilozide on the progression of early carotid wall changes (INSIGHT). Circulation 2001; 103: 2949-54.

41. Borhani NO, Mercuri M, Borhani PA, et al. Final outcome results of the Multicenter Isradipine Diuretic Ather- 
osclerosis Study (MIDAS): a randomized controlled trial. JAMA 1996; 276: 785-791.

42. Zanchetti A, Agabiti-Rosei E, Dal Palù C, et al. The Verapamil in Hypertension ana Atherosclerosis Study (VHAS): results of long-term randomized treatment with either verapamil or chlortalidone on carotid intima-media thickness. J Hypertens 1998; 16: 1667-76.

43. Pitt B, Byington RP, Furberg CD, et al. Effect of amlodipine on the progression of atherosclerosis and the occurrence of clinical events (PREVENT). Circulation 2002; 102: 1503-1510.

44. Stanton AV, Chapman JN, Mayet J, et al. Effects of blood pressure lowering with amlodipine or lisinopril on vascular structure of the common carotid artery. Clin Sci 2001; 101: 455-64.

45. Zanchetti A, Bond MG, Hennig M, et al. Absolute and relative changes in carotid intima-media thickness and atherosclerotic plaques during long-term antihypertensive treatment: further results of the European Lacidipine Study on Atherosclerosis (ELSA). J Hypertens 2004; 22: 1201-12.

46. Rimm EB, Stampfer MJ, Ascherio A, et al. Vitamin E consumption and the risk of coronary heart disease in men. N Engl J Med 1993; 328: 1450-6.

47. Stampfer MJ, Hennekens CH, Manson JE, et al. Vitamin $\mathrm{E}$ consumption and the risk of coronary disease in women. N Engl J Med 1993; 328: 1444-9.

48. Heart Protection Study Collaborative Group. MRC/BHF Heart Protection Study of antioxidant vitamin supplementation in 20536 high-risk individuals: a randomized placebo-controlled trial. Lancet 2002; 360: 7-22.

49. Mc Quillan BM, Hung J, Beilby JP, Nidorf M, Thompson PL. Antioxidant vitamins and the risk of carotid atherosclerosis. The Perth Carotid Ultrasound Disease Assessment Study (CUDAS). J Am Coll Cardiol 2001; 38: 1788-94.

50. Salonen RM, Nyyssonen K, Kaikkonen J, et al. Six-year effect of combined vitamin $\mathrm{C}$ and $\mathrm{E}$ supplementation on atherosclerotic progression. The antioxidant supplementation in atherosclerosis prevention (ASAP) study. Circulation 2003; 107: 947-953.

51. Sawayama Y, Shimizu C, Maeda N, et al. Effects of probucol and pravastatin on common carotid atherosclerosis in patients with asymptomatic hypercholesterolemia. Fukuoka Atherosclerosis Trial (FAST). J Am Coll Cardiol 2002; 39: 610-6.

52. Grayston JT. Antibiotic treatment of atherosclerotic cardiovascular disease. Circulation 2003; 107: 1228-30.

53. Mosorin M, Juvonen J, Biancari F, et al. Use of doxycycline to decrease the growth rate of abdominal aortic aneurysms: a randomized, double-blind, placebo-controlled pilot study. J Vasc Surg 2001; 34: 606-10.

54. Vammen S, Lindholt JS, Ostergaard L, et al. Randomized double-blind controlled trial of roxithromycin for prevention of abdominal aortic aneurysm expansion. Br J Surg 2001; 88: 1066-72.

55. Sander D, Winbeck K, Klingelhofer J, Etgen T, Conrad B. Reduced progression of early carotid atherosclerosis after antibiotic tratment and Chlamydia pneumoniae seropositivity. Circulation 2002; 106: 2428-33.

56. Sander D, Winbeck K, Klingelhofer, J Etgen T, Conrad B. Progression of early carotid atherosclerosis is only temporarily reduced after antibiotic treatment of Chlamydia pneumoniae seropositivity. Circulation 2004; 109: 1010-15.

57. Folsom AR, Eckfeldt JH, Weitzman S, et al. Relation of carotid artery wall thickness to diabetes mellitus, fasting glucose and insulin, body size and physical activity. Atherosclerosis Risk in Communities (ARIC) Study Investigators. Stroke 1994; 25: 66-73.

58. Ebrahim S, Papacosta O, Whincup P, et al. Carotid plaque, intima-media thickness, cardiovascular risk factors and prevalent cardiovascular disease in men and women: the British Regional Heart Study. Stroke 1999; 30: 841-50.

59. Lakka T, Laukkanen J, Rauramaa R, et al. Cardiorespiratory fitness and the progression of carotid atherosclerosis in middle-aged men. Ann Intern Med 2001; 134: 12-20.

60. Nordstrom CK, Dwyer KM, Bairey Merz CN, Shircore A, Dwyer J. Leisure time physical activity and early atherosclerosis: the Los Angeles Atherosclerosis Study. Am J Med 2003; 115: 19-25.

61. Rauramaa R, Halonen P, Valsanen SB, et al. Effects of aerobic physical exercise on inflammation and atherosclerosis in men: the DNASCO Study. Ann Intern Med 2004; 140: 1007-14.

62. Markus RA, Mack WJ, Azen SP, Hodis HN. Influence of lifestyle modification on atherosclerotic progression determined by ultrasonographic change in the common carotid intima-media thickness. Am J Clin Nutr 1997; 65: 1000-4.

63. Okada K, Maeda N, Tatsukawa M, Shimizu C, Sawayama Y, Hayashi J. The influence of lifestyle modification on carotid artery intima-media thickness in a suburban Japanese population. Atherosclerosis 2004; 173: 329-37.

64. Wildman RP, Schott LL, Brockwell S, Kuller LH, SuttonTyrrel K. A dietary and exercise intervention slows menopause-associated progression of subclinical atherosclerosis as measured by intima-media thickness of the carotid arteries. J Am Coll Cardiol 2004; 44: 579-85.

65. Baigent C, Keech A, Kearney PM, et al. Efficacy and safety of cholesterol-lowering treatment: prospective meta-analysis of data from 90,056 participants in 14 randomised trials of statins. Lancet 2005; 366: 1267-78.

66. Sever PS, Dahlof B, Poulter NR, et al. Prevention of coronary and stroke events with atorvastatin in hypertensive patients who have average or lower-than-average cholesterol concentrations, in the Anglo-Scandinavian Cardiac Outcomes Trial-Lipid Lowering Arm (ASCOT-LLA): a muticentre randomised controlled trial. Lancet 2003; 361: 1149-58

67. Collins R, Armitage J, Parish S, et al. Heart Protection Study Collaborative Group. Effects of cholesterol-lowering with simvastatin on stroke and other major vascular events in 20536 people with cerebrovascular disease or other high-risk conditions. Lancet 2004; 363: 757-67. 